\title{
Evaluation of a new disease-specific endovascular device for type B aortic dissection
}

\author{
Germano Melissano, MD, Luca Bertoglio, MD, Andrea Kahlberg, MD, Domenico Baccellieri, MD, \\ Massimiliano M. Marrocco-Trischitta, MD, Fabio Calliari, MD, and Roberto Chiesa, MD
}

From the Chair of Vascular Surgery, "VitaSalute" University, Scientific Institute H. San Raffaele, Milan, Italy.

Received for publication March 14, 2008; revisions received April 30, 2008; accepted for publication June 11, 2008.

Address for reprints: Germano Melissano, MD, IRCCS H. San Raffaele, Department of Vascular Surgery, Via Olgettina, 60, 20132 Milan, Italy (E-mail: g.melissano@ hsr.it).

J Thorac Cardiovasc Surg 2008;136:1012-8 $0022-5223 / \$ 34.00$

Copyright $\odot 2008$ by The American Association for Thoracic Surgery

doi:10.1016/j.jtcvs.2008.06.021
Objective: The study objective was to evaluate the feasibility, safety, and early technical and clinical success rate of a new endovascular device specifically designed for aortic dissection that has recently become available in Europe.

Methods: From June of 2005 to the present, the Zenith Dissection Endovascular System (William Cook Europe, Bjaerverskov, Denmark) was used in 11 selected patients (all male, with a median age of 58 years [range, 45-76 years]) with type B chronic aortic dissection with a compression or collapse of the true lumen. All procedures were performed under general anesthesia with preoperative cerebrospinal fluid drainage in 4 patients. One-step open surgical supra-aortic vessels re-routing was performed in 6 patients to obtain an adequate proximal landing zone: Left carotid-subclavian artery bypass was performed in 5 patients, and right-to-left common carotid artery bypass and left subclavian to common carotid artery transposition was performed in 1 patient. Clinical follow-up visits and computed tomography scans were obtained at 1,6 , and 12 months, and yearly thereafter.

Results: A secondary technical success was obtained in all patients (100\%), and 30day clinical success was achieved in 10 patients (91\%). A type IA entry flow was observed in 1 patient. No mortality was recorded. Occlusion of visceral/renal arteries, retrograde dissections, and device-induced tears in the intimal lamellae were not observed. Periprocedural morbidity included temporary renal failure in 1 patient and postimplantation syndrome with fever and leukocytosis for 23 days in 1 patient. No cases of paraplegia were recorded. At a median follow-up of 12 months (range, 230 months), we observed a clinical success rate of $91 \%$. No migration of the device was observed. No late occlusion of the visceral or renal arteries was recorded at follow-up.

Conclusion: The perioperative and short-term follow-up results showed that the Zenith Dissection Endovascular System for the treatment of aortic dissection can be safely used without affecting the patency of the branches covered by the bare stent. However, these results need to be validated in a larger group of patients with a mid-term follow-up.

$\mathrm{E}$ xisting stent graft technology was developed for treatment of aneurysmatic disease. In the thoracic aorta it should important benefits due to its reduced invasiveness and over the years it has been used also to treat different diseases such as traumatic transection, intramural hematoma, penetrating aortic ulcer and especially aortic dissection. ${ }^{1-15}$

The goal of stent-grafts in aneurysmal disease is to exclude the sac from arterial pressurization to reduce the risk of rupture. However, aortic aneurysms currently represent only $60 \%$ of cases of the indications for thoracic endovascular aortic repair. ${ }^{16}$ For aortic dissection, on the other hand, the objectives are less clear-cut; remodeling and complete healing of the dissected aorta may be obtained in only a fraction of cases. Accepted goals for endovascular treatment of aortic dissections are the following: closure of the primary intimal entry tear, depressurization and thrombosis of the 


\section{Abbreviations and Acronyms \\ $\mathrm{CT}=$ computed tomography \\ PLZ = proximal landing zone \\ TEE $=$ transesophageal echocardiography}

false lumen, and expansion of the true lumen. ${ }^{16}$ To achieve the latter end points and avoid further aneurysmal evolution of the false lumen, stabilization of the intimal flap may play an important role. ${ }^{17}$ The movements of the intimal flap and the continuous motion of the blood in the false lumen clearly contribute in preventing its thrombosis.

This study evaluates the Zenith Dissection Endovascular System (William Cook Europe, Bjaerverskov, Denmark), which uses a series of low radial force bare stents positioned in the aorta distal to the standard stent-graft that covers the primary tear. This device is specifically designed to expand the true lumen, stabilizing the intimal flap, and may be positioned over the origin of the splanchnic and renal vessels.

\section{Materials and Methods}

\section{Patients}

From June of 2005 to the present, the Zenith Dissection Endovascular System was used in 11 nonconsecutive patients with type B chronic aortic dissection. Indications were transaortic growth more than $5 \mathrm{~mm}$ within 6 months or transaortic diameter more than $50 \mathrm{~mm}$ with a compression and collapse of the true lumen. All patients were male with a median age of 58 years (range 4576 years) (Table 1). Patients' demographics and risk factors are reported in Table 2. The median maximum transaortic diameter was $55 \mathrm{~mm}$ (range 48-71 $\mathrm{mm}$ ). The median interval between the clinical presentation of the type $\mathrm{B}$ dissection and the endovascular procedure was 19 months (range 3-29 months).

\section{Device Description}

The Zenith Dissection Endovascular System is a modular system that is specifically designed to treat aortic dissection and that consists of a proximal component, the Zenith TX2-P TAA Endovascular Graft, and a distal component, the Zenith Dissection Endovascular stent (Figure 1).

The proximal component is a 1-piece (cylindric or tapered) endovascular stent-graft that is intended to close the primary entry tear. The stent-graft is fully stented, covered with polyester fabric, and contains protruding barbs at a 2-mm stagger at its proximal end and an internal sealing stent without barbs at its distal end. No proximal or distal bare stent is present. The stent-graft component of the Zenith Dissection Endovascular System is the same as the proximal component of the 2-piece system also used for treatment of thoracic aneurysms. The Zenith TX2-P TAA Endovascular Graft is Conformité Européenne marked and received approval from the Food and Drug Administration in the United States in May 2008. ${ }^{18}$

The distal component is a 1-piece cylindric device made from self-expanding, stainless-steel Cook Z-stent segments sewn together with polyester suture and no covering fabric. It is intended to provide support to the delaminated segments of the dissected aorta, and the stent has a lower radial force than the TX2 stent-graft. The Zenith Dissection Endovascular stent is available in only 1 diameter $(46 \mathrm{~mm})$ fitting all the aortic diameters and different lengths. It is currently supplied as a custom-made device.

\section{Methods}

The feasibility of the endovascular procedure and sizing of the stentgrafts were determined with 64-row multislice computed tomography with multiplanar reconstructions. Diagnostic workup included duplex scanning of the iliac, femoral, carotid, and vertebral arteries to evaluate the adequacy of remote arterial access sites and assess the patency of extracranial supra-aortic trunks.

Anatomic endovascular inclusion criteria were as follows:

- primary entry tear more than $20 \mathrm{~mm}$ below the innominate artery (covering the left subclavian artery or the left common

TABLE 1. The Zenith Dissection Endovascular System (William Cook Europe, Bjaerverskov, Denmark) used and supra-aortic vessels re-routing open procedures

\begin{tabular}{|c|c|c|c|c|c|}
\hline $\mathbf{N}$ & Sex/age $(y)$ & Dissection type & Proximal endograft & Distal bare stent & SAT debranching \\
\hline 1 & $\mathrm{M} / 47$ & Post type A repair & ZTEG 2PT $34 \times 197$ & GZSD $46 \times 123$ & Right to left CCB + left SCT \\
\hline 2 & $\mathrm{M} / 61$ & Chronic type B & ZTEG 2 P $36 \times 127$ & GZSD $46 \times 164$ & Left CSB \\
\hline 3 & $\mathrm{M} / 47$ & Chronic type B & ZTEG 2PT $38 \times 152$ & GZSD $46 \times 164$ & Bilateral CSB \\
\hline 4 & $\mathrm{M} / 45$ & Subacute type B & ZTEG 2 P $32 \times 200$ & $\begin{array}{l}\text { GZSD } 46 \times 82 \\
\text { GZSD } 46 \times 164\end{array}$ & None \\
\hline 5 & $\mathrm{M} / 72$ & Chronic type B & $\begin{array}{l}\text { ZTEG } 2 \text { P } 30 \times 140 \\
\text { ZTEG } 2 \text { PT } 34 \times 157\end{array}$ & GZSD $46 \times 164$ & None \\
\hline 6 & $\mathrm{M} / 76$ & Chronic type B & ZTEG 2P $36 \times 157$ & GZSD $46 \times 164$ & Left CSB \\
\hline 7 & $\mathrm{M} / 58$ & Chronic type B & ZTEG 2 PT $36 \times 157$ & GZSD $46 \times 164$ & Left CSB \\
\hline 8 & $\mathrm{M} / 53$ & Chronic type B & ZTEG 2 PT $42 \times 158$ & GZSD $46 \times 123$ & None \\
\hline 9 & $\mathrm{M} / 65$ & Subacute type B & ZTEG 2PT $34 \times 157$ & GZSD $46 \times 164$ & Left CSB \\
\hline 10 & $\mathrm{M} / 64$ & Chronic type B & ZTEG 2P $28 \times 152$ & GZSD $46 \times 164$ & None \\
\hline 11 & $\mathrm{M} / 54$ & Chronic type B & ZTEG 2PT $36 \times 197$ & GZSD $46 \times 123$ & None \\
\hline
\end{tabular}

SAT, Supra-aortic trunk; ZTEG, Zenith thoracic endograft; 2P, TX2 proximal cylindrical; 2PT, TX2 proximal tapered; GZSD: Gianturco Z-stent dissection, CCB, carotid-to-carotid bypass; $S C T$, subclavian to carotid transposition, $C S B$, carotid to subclavian bypass. 
TABLE 2. Patients' demographics and risk factors

\begin{tabular}{lc}
\hline \multicolumn{1}{c}{ Risk factors } & N \\
\hline Median age & 54 y \\
Sex (men) & 11 \\
Tobacco use & 6 \\
Diabetes & 1 \\
Hypertension & 9 \\
Pulmonary disease & 2 \\
Renal disease & 1 \\
Cardiac disease & 3 \\
Cerebrovascular disease & 0 \\
Previous ascending aorta repair & 3 \\
\hline
\end{tabular}

carotid artery was considered acceptable with previous debranching and revascularization);

- primary entry tear more than $20 \mathrm{~mm}$ above celiac trunk;

- proximal landing zone (PLZ) diameter for stent-graft, measured from outer wall to outer wall on a sectional image or multiplanar reconstruction more than $24 \mathrm{~mm}$ and less than $38 \mathrm{~mm}$, respectively;

- distal landing zone diameter for stent-graft, measured outer wall to outer wall on a sectional image or multiplanar reconstruction more than $24 \mathrm{~mm}$ (estimate based on transaortic diameter) or less than $38 \mathrm{~mm}$ (estimate based on true lumen diameter); and

- any segment of vessel into which deployment of bare stent device is intended measuring more than $24 \mathrm{~mm}$ (estimate based on transaortic diameter) or less than $38 \mathrm{~mm}$ (estimate based on true lumen diameter).

All procedures were performed in the operating room under general anesthesia, using a portable digital C-arm image intensifier and intraoperative transesophageal echocardiography (TEE). Preoperative cerebrospinal fluid drainage was used in 4 patients. In 6 patients, supra-aortic vessels re-routing was performed before the stent-graft deployment to obtain an adequate PLZ: 4 cases of left carotid-subclavian artery bypass for zone 2 PLZ, 1 case of bilateral carotid-subclavian artery bypass for right aberrant subcla- vian artery, and 1 case of right to left common carotid artery and left subclavian to common carotid artery transposition for zone 1 PLZ (Table 1).

The common femoral artery, exposed through an inguinal incision, was used as the remote access vessel in all patients. An initial aortography to assess the patency of visceral vessels and their origin from the true and false lumens was performed. A superstiff Lunderquist precurved guidewire (Cook Inc, Bloomington, Ind) was placed over a pigtail catheter navigated in the true lumen, under both fluoroscopic and TEE guidance, to ensure definite positioning of the stiff wire in the true lumen, which is essential for correct deployment of the stent-graft. The Zenith TX2-P stent-graft (Table 1) was carefully advanced over the stiff wire and deployed in the intended position. Aortography and TEE were then performed to ensure correct exclusion of proximal entry tear. The Zenith Dissection Endovascular Stent (Table 1) and its delivery system were then inserted through the same sheath and wire used for deployment of the proximal component. The stent was then advanced in the planned location and deployed overlapping 1 stent with the proximal stent-graft. Ballooning of the bare stent device was not performed. Final angiography and TEE completed the procedure. Careful angiographic evaluation of the patency of visceral and renal arteries was performed in all patients.

\section{Success Measures, Follow-up Protocol, and Statistical Analysis}

The reported results and methods are in accordance with the current reporting standards for endovascular aortic repair prepared and revised by the Ad Hoc Committee for Standardized Reporting Practices in Vascular Surgery (Society for Vascular Surgery/American Association for Vascular Surgery). ${ }^{19}$ Patients were evaluated with postprocedure chest $\mathrm{x}$-ray and contrast computed tomography (CT) scans with scheduled follow-up imaging at 1, 6, and 12 months, and yearly thereafter. Angiograms were obtained in selected cases. Clinical follow-up was also done at regular intervals of 6 months. Data are shown as number (\%) for categoric variables or as median, first quartile, and third quartile (Q1-Q3) and mean for continuous variables, because they did not show a Gaussian distribution. Analyses were performed with SAS v8 software (SAS Institute Inc, Cary, NC).
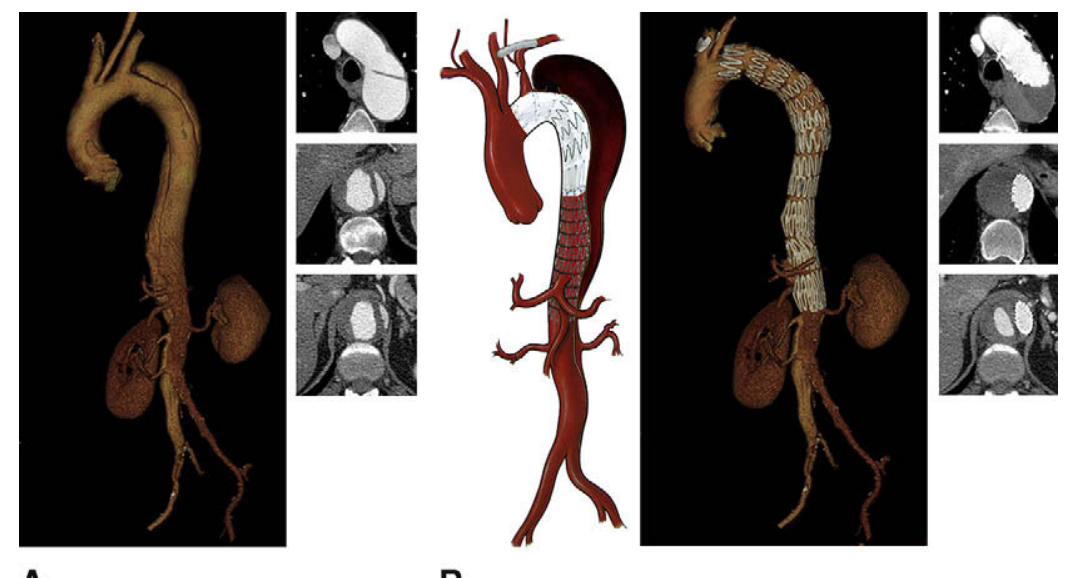

Figure 1. A, Preoperative 3-dimensional CT scan of a case of type $B$ aortic dissection with proximal neck located on zone 2. B, Postoperative 3-dimensional CT scan after a left carotid-subclavian artery bypass and successful deployment of the Zenith Dissection Endovascular System (William Cook Europe, Bjaerverskov, Denmark). Note the complete thrombosis of proximal and mid-thoracic false lumens with reexpansion of the true lumen and reperfusion of the distal thoracic false lumen because of presence of reentry tears. 


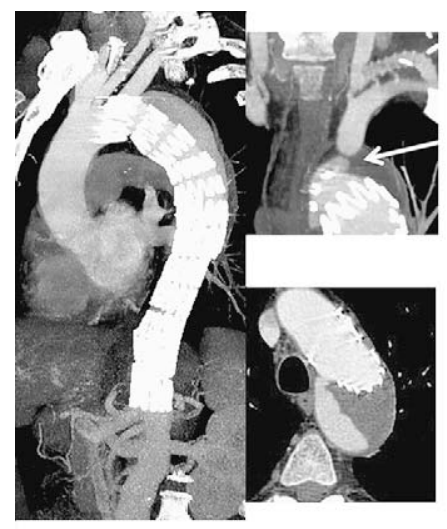

A

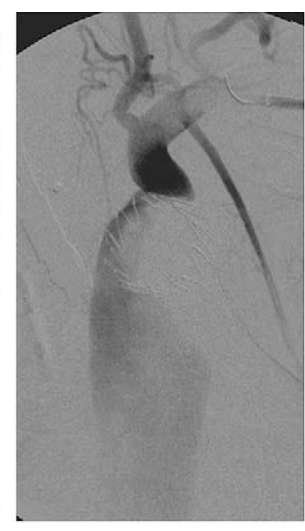

B

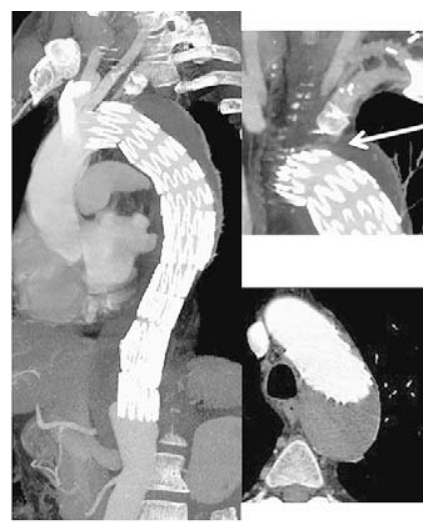

C
Figure 2. A, One-month postoperative CT scan of a case of type B aortic dissection showing a type II reentry flow from a previous revascularized left subclavian artery (arrow). B, Retrograde angiogram from the left subclavian artery showing complete false lumen perfusion. C, Predischarge CT scan after procedure of endovascular occlusion of the origin of the left subclavian artery with an Amplatzer plug (AGA Medical, Golden Valley, MN, USA) showing complete thrombosis of false lumen with resolution of the type II reentry flow.

\section{Results}

The primary entry tear was located just distal to the subclavian artery in 9 cases, in one case it was located at the $2 \mathrm{~mm}$ above the left subclavian artery and in the case of right aberrant subclavian artery the vessel origin was directly involved by the dissection. The dissection extended down into 1 ( 9 cases) or both ( 2 cases) iliac arteries. The false lumen was patent in all cases. The branch vessels were compromised by an exclusively dynamic process in all cases, and no branch vessels originated from the false lumen. The median diameter of the landing zone for the Zenith TX2-P TAA Endovascular Graft was $32 \mathrm{~mm}$ (range 26-37 mm) proximally and $22 \mathrm{~mm}$ (range 16-27 $\mathrm{mm}$ ) distally, estimated on the basis of the true lumen diameter. The smaller segment of vessel into which the deployment of the bare stent was performed was $14 \mathrm{~mm}$ (range 12-24 mm), estimated on the basis of the true lumen diameter.

TEE evaluation performed 10 to 15 minutes after Zenith TX2 TAA Endovascular Graft deployment showed a complete spontaneous echo-contrast effect, the so-called "smoke" phenomenon, ${ }^{20}$ in 9 patients $(82 \%)$. In 1 patient, persistent perfusion of the false lumen caused by type IA endoleak was successfully treated with proximal ballooning. In 1 patient, a type IB peri-stent leak with a pulsed Doppler velocity greater than $50 \mathrm{~cm} / \mathrm{sec}$ caused by a distal reentry tear was treated with an adjunctive Zenith TX2-P stent-graft. In all patients, completion angiography demonstrated complete exclusion of the proximal entry tear and TEE showed complete spontaneous echo-contrast effect, with a rate of secondary technical success of $100 \%$. No occlusion of the visceral or renal artery was recorded. No additional surgical or endovascular procedures were necessary.

No 30-day mortality was recorded in this series. The median intensive care unit stay was 1 day (range 1-2 days). Clinical success was observed in 10 patients $(91 \%) ; 1$ case of type IA entry flow was observed at predischarge CT scan. Left subclavian artery percutaneous occlusion was per- formed as an adjunctive procedure to correct a type II entry flow in 1 patient (Figure 2). A type II entry flow from an intercostal artery was observed in 1 patient, who was treated conservatively.

Periprocedural morbidity (30-day) included 1 case of serum creatinine increase more than $30 \%$ versus the preprocedure values and 1 case of postimplantation syndrome. The patient had fever, leukocytosis, and increased biochemical inflammatory markers without evidence of sepsis or infection, which resolved after corticosteroids administration on the 24th postoperative day. No cases of paraplegia were recorded. The median in-hospital stay was 4 days (range $2-$ 6 days).

At a median follow-up of 11.7 months (range 2-30 months), we observed a clinical success rate of $91 \%$ (10/11 cases): spontaneous resolution of the procedural type I entry flow and 1 case of false lumen enlargement of $4 \mathrm{~mm}$. No migration of the Zenith Dissection Endovascular System was observed. No occlusion of the visceral or renal arteries was recorded.

The false lumen showed different characteristics in the thoracic segment than in the celiac segment. Where the Zenith TX2 TAA Endovascular Graft was deployed, thrombosis of the false lumen was observed in all patients, with shrinkage of the false lumen in 4 patients, no change in 6 patients, and false lumen enlargement of $4 \mathrm{~mm}$ in 1 patient. Where the Zenith Dissection Endovascular stent was deployed, false lumen thrombosis was not observed and there was no shrinkage ( $>5 \mathrm{~mm}$ ). However, in all cases, a median reexpansion of the true lumen of $5 \mathrm{~mm}$ (range 2-7 $\mathrm{mm}$ ) without any change of the transaortic diameter was observed.

\section{Discussion}

Stent-graft repair of descending thoracic aortic aneurysms currently offers encouraging outcomes when compared with those of open surgery. ${ }^{1,2,4}$ Stent-grafts have been used to treat dissections since the 1990s; however, the effectiveness of this procedure remains unproven for patients with 
thoracic aortic aneurysms originating from a chronic type B dissection.

The rationale of endovascular treatment for aortic dissection is based on the exclusion of the false lumen from the circulation. In chronic dissections, the graft does not immediately compress the dissection membrane against the aortic wall as in acute dissections. However, the closure of the primary entry tear redirects the blood flow into the true lumen, thus decompressing the false lumen. As a result of the effective obliteration of the primary entry tear, a state of low turbulent flow in the thoracic portion of the false lumen was evident in all our patients at TEE. Despite heparinization, initial thrombosis was observed in 9 patients and postoperative CT showed thrombosis of the proximal descending thoracic false lumen in 10 patients.

Complete healing of a chronic dissection may be expected in only a fraction of cases; healing requires some time and has relevant prognostic consequences. Recent insights on the natural history of chronic dissections showed partial thrombosis of the false lumen to be a strong independent predictor of mortality. ${ }^{21}$ This may be related to an increased pressure in the false lumen, secondary to the occlusion of distal tears that impedes outflow, resulting in a blind sac that may cause further aneurysmal dilatation, re-dissection, or rupture.

Accepted goals of simple exclusion of proximal entry tear are decompression of the thoracic false lumen and promotion of proximal thrombosis and remodeling of the false lumen. However, the distal thoracic and abdominal dissected aortas fails to remodel in $50 \%-80 \%$ of cases, ${ }^{15}$ probably due to both distal communication sites between the true and false lumens and the flapping motion of the dissecting lamella, which prevents complete false lumen thrombosis. Aortic remodeling may fail with this limited approach with consequent risk of further aneurysmal expansion, extension of the dissection, or rupture. Aggressive management of distal thoracic entry tears by means of adjunctive stent-grafts deployment above the celiac trunk could help to enhance false lumen thrombosis. However, the risk of spinal cord ischemia increases with a stent-graft coverage longer than $20 \mathrm{~cm} .{ }^{22}$ Moreover, true lumen compression at the level of the abdominal aorta and consequent visceral branch malperfusion are not fully addressed with this approach.

Ito and colleagues ${ }^{23}$ reported a case of type B aortic dissection with malperfusion that was successfully treated with implantation of a Gianturco Z-stent (Cook, Inc., Bloomington, IN) in the distal thoracic and abdominal aorta. Nienaber and colleagues ${ }^{17}$ proposed the Provisional Extension to Induce Complete Attachment (the PETTICOAT Concept) to treat distal true lumen collapse and a perfused abdominal false lumen despite successful stent-graft sealing of the proximal tears. The authors successfully placed a bare metal scaffolding stent for distal extension of the previously implanted stent-graft (as an adjunctive or staged procedure) in 12 patients, achieving the re-expansion of the true lumen and resolution of malperfusion in all cases. In this series, sinus aortic stent (Optimed Medizinische Instrumente GmbH., Ettlingen, Germany) and Fortress stents (Curative Medical Devices Inc., Dresden Germany) were used in 11 patients and the Zenith Dissection Endovascular stent was used in 1 patient.

Mossop and colleagues ${ }^{24}$ recently reported the treatment of 7 patients with type B acute aortic dissection with stentgraft (Zenith TX2 TAA Endovascular Graft) closure of the proximal entry tear and a bare metal Z-stent deployed in the residual delaminated aorta. They used Gianturco Z-stents in 3 patients and the Zenith Dissection Endovascular stent in 4 patients, achieving a mean immediate abdominal true lumen expansion greater than $130 \%$ in all patients. No long-term adverse events related to Z-stent implantation, including stent migration, fracture, intimal flap erosion, or redissection, were reported.

The possible detrimental effects of bare stent struts extended to or above the level of renal or visceral arteries have been investigated for endovascular treatment of infrarenal aortic aneurysms with stent-graft with suprarenal fixation. The majority of these studies observed no adverse effect of suprarenal fixation on renal function at mid-term follow-up and no visceral artery perfusion impairment. ${ }^{25,26}$

In our study, we used the complete Zenith Dissection Endovascular system in all patients. At follow-up, we observed a successful proximal thrombosis of the false lumen in all patients and enlargement at 12 months despite exclusion of the false lumen in 1 patient (9\%). No adverse events related to the Zenith Dissection Endovascular Stent were observed. A median rate of reexpansion of the true lumen of the distal thoracic and abdominal aorta of $5 \mathrm{~mm}$ was detected. Mossop and colleagues ${ }^{24}$ reported an increase in the mean abdominal aortic true lumen index (true lumen area/total aorta area) from $41 \% \pm 17 \%$ (standard deviation) to $75 \% \pm 14 \%$ (standard deviation) with the Zenith Dissection Endovascular stent. The lower rate of re-expansion reported may be explained by the different timing of treatment of the aortic dissection in our study (chronic vs acute).

Retrograde type A dissection is a potentially lethal complication of endovascular stent-graft repair that may occur as immediate or delayed. ${ }^{27,28}$ In our previous experience, an acute retrograde dissection developed in a patient 3 days after undergoing endovascular repair of an acute type B dissection with a proximal bare stent endograft, leading to myocardial infarction, pericardial tamponade, and death. ${ }^{29}$ Several factors are believed to play a role in the development of such a complication. ${ }^{30}$ In particular, the presence of proximal uncovered bare stents or bare springs with high radial force may lead to an injury of the intima. ${ }^{31,32}$

The proximal component of the Zenith Dissection Endovascular System used in our study contains protruding barbs at a 2-mm stagger at its proximal end without a proximal bare stent. In our experience, we did not observe retrograde 
dissection using this device. The distal end of the stent-graft has an internal sealing stent without barbs, as well as the Zenith Dissection Endovascular Stent. This peculiar characteristic is important to avoid damage of the dissecting lamella in the distal descending thoracic and abdominal aorta. Moreover, a tapered proximal stent-graft reduces the oversize in the dissected aorta and avoids device-induced tears or dissection.

Endovascular stent-grafting has recently become an alternative treatment option in patients with type B aortic dissections. Recent reports confirmed the safety and efficacy in terms of 1-year survival in comparison with medical therapy and surgery. ${ }^{33}$ The effectiveness of endovascular therapy is secondary to reduced procedure time, low rate of bleeding, intraoperative complications, and lower associated morbidity. The final objective of stent grafting is represented by depressurization with thrombosis of the false lumen. Patency of the false lumen was reported to be a predictor of outcome in patients with type B aortic dissection. Several studies have suggested an improved outcome in patients with complete thrombosis of the false lumen and an increased risk of complications and death because of aortic expansion in patients with a patent false lumen. ${ }^{34}$

\section{Conclusions}

Our perioperative and short-term follow-up results with the use of the Zenith Dissection Endovascular System device for the treatment of aortic dissection in a small group of selected patients showed that, in this clinical setting, the device can be safely inserted without significantly modifying the operating time and morbidity or affecting the patency of the branches that were covered by the bare stents. All patients survived; a stable or improved situation of the dissected aorta was achieved in 10 of 11 patients. The continued need for surveillance is obviously still mandatory. Also, preliminary results in different clinical settings should be evaluated before a more widespread use of the device can be suggested.

We acknowledge Dr Giuseppe Crescenzi, from the Department of Cardiovascular Anesthesia and Intensive Care of San Raffaele Hospital, for performing TEE evaluation during all the procedures of the study, and Dr Gliliola Calori, from the Statistical Unit of San Raffaele Hospital, for help with the statistical analysis of the article's data.

\section{References}

1. Makaroun MS, Dillavou ED, Kee ST, Sicard G, Chaikof E, Bavaria J, et al. Endovascular treatment of thoracic aortic aneurysms: results of the phase II multicenter trial of the GORE TAG thoracic endoprosthesis. $J$ Vasc Surg. 2005;41:1-9.

2. Bavaria JE, Appoo JJ, Makaroun MS, Verter J, Yu ZF, Mitchell RS. Gore TAG Investigators. Endovascular stent grafting versus open surgical repair of descending thoracic aortic aneurysms in low-risk patients: a multicenter comparative trial. J Thorac Cardiovasc Surg. 2007;133:369-77.

3. Fattori R, Nienaber CA, Rousseau H, Beregi JP, Heijmen R, Grabenwöger M, et al. Talent Thoracic Retrospective Registry. Results of endovascular repair of the thoracic aorta with the Talent Thoracic stent graft: the Talent Thoracic Retrospective Registry. J Thorac Cardiovasc Surg. 2006;132:332-9.

4. Matsumura JS, Cambria RP, Dake MD, Moore RD, Svensson LG, Snyder S. TX2 Clinical Trial Investigators. International controlled clinical trial of thoracic endovascular aneurysm repair with the Zenith TX2 endovascular graft: 1-year results. J Vasc Surg. 2008;47:247-57.

5. Kato N, Dake MD, Miller DC, Semba CP, Mitchell RS, Razavi MK, et al. Traumatic thoracic aortic aneurysm: treatment with endovascular stent-grafts. Radiology. 1997;205:657-62.

6. Tehrani HY, Peterson BG, Katariya K, Morasch MD, Stevens R, DiLuozzo G, et al. Endovascular repair of thoracic aortic tears. Ann Thorac Surg. 2006;82:873-7.

7. Juszkat R, Pukacki F, Oszkinis G, Zarzecka A, Majewski W. Intramural hematoma of the thoracic aorta as a form of aortic dissection. Cardiovasc Intervent Radiol. 2007;30:1056-60.

8. Sundt TM. Intramural hematoma and penetrating atherosclerotic ulcer of the aorta. Ann Thorac Surg. 2007;83:S835-41.

9. Demers P, Miller DC, Mitchell RS, Kee ST, Chagonjian L, Dake MD. Stent-graft repair of penetrating atherosclerotic ulcers in the descending thoracic aorta: mid-term results. Ann Thorac Surg. 2004;77:81-6.

10. Eggebrecht H, Herold U, Schmermund A, Lind AY, Kuhnt O, Martini S, et al. Endovascular stent-graft treatment of penetrating aortic ulcer: results over a median follow-up of 27 months. Am Heart J. 2006;151:530-6.

11. Dalainas I, Nano G, Medda M, Bianchi P, Casana R, Ramponi F, et al. Endovascular treatment of penetrating aortic ulcers: mid-term results. Eur J Vasc Endovasc Surg. 2007;34:74-8.

12. Dake MD, Kato N, Mitchell RS, Semba CP, Razavi MK, Shimono T, et al. Endovascular stent-graft placement for the treatment of acute aortic dissection. N Engl J Med. 1999;340:1546-52.

13. Nienaber CA, Fattori R, Lund G, Dieckmann C, Wolf W, von Kodolitsch $\mathrm{Y}$, et al. Nonsurgical reconstruction of thoracic aortic dissection by stent-graft placement. N Engl J Med. 1999;340:1539-45.

14. Umaña JP, Miller DC, Mitchell RS. What is the best treatment for patients with acute type B aortic dissections-medical, surgical, or endovascular stent-grafting? Ann Thorac Surg. 2002;74:S1840-3.

15. Eggebrecht $H$, Nienaber $C A$, Neuhäuser $M$, Baumgart $D$, Kische $S$, Schmermund A, et al. Endovascular stent-graft placement in aortic dissection: a meta-analysis. Eur Heart J. 2006;27:489-98.

16. Dake MD, Wang DS. Will stent-graft repair emerge as treatment of choice for acute type B dissection? Semin Vasc Surg. 2006;19:40-7.

17. Nienaber CA, Kische S, Zeller T, Rehders TC, Schneider H, Lorenzen B, et al. Provisional extension to induce complete attachment after stentgraft placement in type B aortic dissection: the PETTICOAT concept. J Endovasc Ther. 2006;13:738-46.

18. Matsumura JS, Cambria RP, Dake MD, Moore RD, Svensson LG, Snyder S. TX2 Clinical Trial Investigators. International controlled clinical trial of thoracic endovascular aneurysm repair with the Zenith TX2 endovascular graft: 1-year results. J Vasc Surg. 2008;47:247-57.

19. Chaikof EL, Blankensteijn JD, Harris PL, White GH, Zarins CK, Bernhard VM, et al. Ad Hoc Committee for Standardized Reporting Practices in Vascular Surgery of The Society for Vascular Surgery/ American Association for Vascular Surgery. Reporting standards for endovascular aortic aneurysm repair. J Vasc Surg. 2002;35:1048-60.

20. Rocchi G, Lofiego C, Biagini E, Piva T, Bracchetti G, Lovato L, et al. Transesophageal echocardiography-guided algorithm for stent-graft implantation in aortic dissection. J Vasc Surg. 2004;40:880-5.

21. Tsai TT, Evangelista A, Nienaber CA, Myrmel T, Meinhardt G, Cooper JV, et al. International Registry of Acute Aortic Dissection. Partial thrombosis of the false lumen in patients with acute type B aortic dissection. N Engl J Med. 2007;357:349-59.

22. Buth J, Harris PL, Hobo R, van Eps R, Cuypers P, Duijm L, Tielbeek X. Neurologic complications associated with endovascular repair of thoracic aortic pathology: incidence and risk factors. a study from the European Collaborators on Stent/Graft Techniques for Aortic Aneurysm Repair (EUROSTAR) registry. J Vasc Surg. 2007;46:1103-10.

23. Ito N, Tsunoda T, Nakamura M, Iijima R, Matsuda K, Suzuki T, et al. Percutaneous bare Z-stent implantation as an alternative to surgery for acute aortic dissection with visceral ischemia. Catheter Cardiovasc Interv. 2003;58:95-100.

24. Mossop P, Nixon I, Oakes J, Devine TJ, McLachlan CS. Immediate "total" aortic true lumen expansion in type A and B acute aortic 
dissection after endovascular aortic endografting and GZSD bare stenting. J Thorac Cardiovasc Surg. 2007;134:1360-2.

25. Walsh SR, Boyle JR, Lynch AG, Sadat U, Carpenter JP, Tang TY, et al. Suprarenal endograft fixation and medium-term renal function: systematic review and meta-analysis. J Vasc Surg. 2008;47:1364-70. Epub 2008 Feb 14.

26. Greenberg RK, Chuter TA, Lawrence-Brown M, Haulon S, Nolte L. Zenith Investigators. Analysis of renal function after aneurysm repair with a device using suprarenal fixation (Zenith AAA Endovascular Graft) in contrast to open surgical repair. J Vasc Surg. 2004;39:1219-28.

27. Pasic M, Bergs P, Knollmann F, Zipfel B, Müller P, Hofmann M, et al. Delayed retrograde aortic dissection after endovascular stenting of the descending thoracic aorta. J Vasc Surg. 2002;36:184-6.

28. Neuhauser B, Czermak BV, Fish J, Perkmann R, Jaschke W, Chemelli A, et al. Type A dissection following endovascular thoracic aortic stent-graft repair. $J$ Endovasc Ther. 2005;12:74-81.

29. Melissano G, Tshomba Y, Civilini E, Chiesa R. Disappointing results with a new commercially available thoracic endograft. J Vasc Surg. 2004;39:124-30.
30. Neuhauser B, Greiner A, Jaschke W, Chemelli A, Fraedrich G. Serious complications following endovascular thoracic aortic stent-graft repair for type B dissection. Eur J Cardiothorac Surg. 2008;33:58-63.

31. Grabenwoger M, Fleck T, Ehrlich M, Czerny M, Hutschala D, Schoder M, et al. Secondary surgical interventions after endovascular stent-grafting of the thoracic aorta. Eur J Cardiothorac Surg. 2004;26: 608-13.

32. Rubin S, Bayle A, Poncet A, Baehrel B. Retrograde aortic dissection after a stent graft repair of a type B dissection: how to improve the endovascular technique. Interact Cardiovasc Thorac Surg. 2006;5: 746-8.

33. Trimarchi S, Nienaber CA, Tsai TT, Llovet A, Froelich J, Rampoldi V. In Search For Optimal Treatment of Complicated Acute Type B Aortic Dissection: Insights from the International Registry of Acute Aortic Dissection (IRAD). Circulation. 2006;114:II432-3.

34. Bernard Y, Zimmermann H, Chocron S, Litzler JF, Kastler B, Etievent JP, et al. False lumen patency as a predictor of late outcome in aortic dissection. Am J Cardiol. 2001;87:1378-82. 